\title{
FREQÜÊNCIA DOS ANTÍGENOS DE HISTOCOMPATIBILIDADE NA POPULAÇÃO NORMAL DA REGIÃO NORDESTE DO ESTADO DE SÃO PAULO BRASIL
}

\author{
FREQUENCY OF HISTOCOMPATIBITY ANTIGENS IN THE NORMAL POPULATION OF THE \\ NORTHEAST REGION OF THE STATE OF SÃO PAULO BRAZIL
}

Eduardo A Donadi ${ }^{1}$; Luís Maurício-da-Silva²; Cássia M Paula-Santos ${ }^{3}$; Rubens D Silveira ${ }^{4}$; Neifi H S Deghaide ${ }^{4}$; Agenor S Ferraz ${ }^{5}$ \& Júlio C Voltarelli ${ }^{1}$

\begin{abstract}
'Docentes do Departamento de Clínica Médica da Divisão de Imunologia Clínica da Faculdade de Medicina de Ribeirão Preto - USP; ${ }^{2}$ Docente do Departamento de Genética do Centro de Ciências Biológicas da Universidade Federal de Pernambuco - UFP, Recife; ${ }^{3}$ Técnico especializado da Faculdade de Medicina de Ribeirão Preto USP; ${ }^{4}$ Técnico especializado do Hospital das Clínicas da Faculdade de Medicina de Ribeirão Preto; ${ }^{5}$ Docente do Departamento de Cirurgia da Faculdade de Medicina de Ribeirão Preto - USP.

CorRespondência: Prof Dr Eduardo A Donadi - Divisão de Imunologia Clínica, Departamento de Clínica Médica, Faculdade de Medicina de Ribeirão Preto - Universidade de São Paulo - 14049 - 900 Ribeirão Preto SP - Fax: 16-633 9995 E-mail: eadonadi@fmrp.usp.br
\end{abstract}

DONADI EA; MAURÍCIO-DA-SILVA L; PAULA-SANTOS CM; SILVEIRA RD; DEGHAIDE NHS; FERRAZ AS \& VOLTARELLI JC. Freqüência dos antígenos de histocompatibilidade na população normal da região nordeste do estado de São Paulo Brasil. Medicina, Ribeirão Preto, 33: 19-26, jan./mar. 2000.

RESUMO: Modelo de estudo: estudo de prevalência.

Objetivos: avaliação das freqüências dos antígenos de histocompatibilidade de classe I (HLA-A, -B e -C) e de classe II (HLA-DR e -DQ) na população de indivíduos saudáveis da região nordeste do Estado de São Paulo.

Metodologia: foram estudados três grupos de indivíduos saudáveis, o de funcionários do Hospital de Clínicas e da Faculdade de Medicina de Ribeirão Preto, o de doadores do Banco de Sangue local e o de doadores cadáveres de órgãos sólidos do São Paulo Interior Transplante. Os antígenos HLA foram tipificados, utilizando-se método de microlinfocitotoxicidade-dependente de complemento.

Resultados: as freqüências dos antígenos de histocompatibilidade de classe I ou II, usualmente detectados entre os três grupos estudados, foram semelhantes.

Conclusões: as populações de indivíduos saudáveis estudadas refletem adequadamente o perfil imunogenético da população da região nordeste do Estado de São Paulo, podendo esses dados ser utilizados para estudos de seleção de doadores em transplantes, e, também, naqueles de associação com doenças.

UNITERMOS: Antígenos HLA. Sorologia. Antígenos de Histocompatibilidade Classe I. Antígenos de Histocompatibilidade Classe II.

\section{INTRODUÇÃO}

Os estudos relacionados com a associação dos antígenos de histocompatibilidade (HLA) e doenças baseiam-se, fundamentalmente, nas comparações das freqüências desses marcadores entre pacientes e indivíduos saudáveis, utilizados como controles. Uma vez que os genes que codificam as moléculas HLA apresentam um polimorfismo muito grande, nas diversas populações estudadas, a definição do perfil imunogenético da população de indivíduos saudáveis de cada região é essencial para esses estudos. Mesmo em indivíduos caucasianos da América do Norte ou da Europa, o número de especificidades dos loci HLA-A, -B, -C e 
das regiões HLA-DR e -DQ é significativamente grande, quando comparado, por exemplo, com o dos indivíduos nativos ou com os dos aborígenes da América Latina $^{(1,2)}$. Nesse aspecto, na população brasileira, caracterizada pela alta taxa de miscigenação entre caucasianos, africanos e índios, dever-se-ia esperar uma diversidade ainda maior dos antígenos e alelos de histocompatibilidade. Realmente, os poucos estudos imunogenéticos existentes, utilizando-se métodos sorológicos ou moleculares para identificação das especificidades HLA, corroboram essa idéia ${ }^{(3 / 8)}$. Assim, para estudarmos o tema associação de antígenos HLA com as doenças, a caracterização dos indivíduos normais da nossa região é fundamental. Neste estudo, estamos relatando as frequiências dos antígenos HLA de classe I e II em três populações de indivíduos saudáveis, procedentes da região nordeste do Estado de São Paulo.

\section{MATERIAL E MÉTODOS}

\section{Indivíduos normais}

Para o estudo das freqüências dos antígenos de histocompatibilidade de classe I e II foram estudados três grupos. O grupo número 1 (G1) era constituído por 100 indivíduos saudáveis, todos caucasóides, não aparentados, pertencentes ao corpo de funcionários do Hospital das Clínicas e da Faculdade de Medicina de Ribeirão Preto, da Universidade de São Paulo. O grupo de número 2 (G2) era constituído por 257 doadores cadáveres de órgãos sólidos, provenientes do São Paulo Interior Transplante (SPIT), considerados normais até 24 horas antes de suas mortes, sendo 212 caucasóides, 31 mulatos, 12 negros e 02 amarelos. O grupo de número 3 (G3) era composto por 191 doadores voluntários do Banco de Sangue do Hospital das Clínicas da Faculdade de Medicina de Ribeirão Preto, sendo 151 caucasóides, 23 mulatos e 17 negros.

\subsection{Tipificação dos antígenos de histocompati- bilidade}

As células linfomononucleares do sangue periférico foram isoladas em gradiente de Ficoll-Hypaque, densidade $1077 \mathrm{~g} / \mathrm{l}$. Os linfócitos B foram obtidos, utilizando-se lã de "nylon" (Robbins Scientific, EUA). As especificidades HLA foram tipificadas usando-se teste de microlinfocitotoxicidade-dependente de complemento ${ }^{(9)}$. Os seguintes anti-soros foram utilizados: HLA-A (A1, A2, A3, A9, A10, A11, A19, A23, A24, A25, A26, A28, A29, A30, A31, A33, A34, A36); HLA-B (B5, B7, B8, B12, B13, B14, B15,B16, B17, B18, B21, B22, B27, B35, B37, B38, B39, B40, B41,
B42, B44, B45, B47,B48, B49, B50, B51, B52, B54, B55, B56, B60, B61, B64, B65, B70), HLA-Bw4, -Bw6; HLA-C (Cw1- Cw8); HLA-DR (DR1, DR2, DR3, DR4, DR5, DR6, DR7, DR8, DR9, DR10), HLA-DR52 e HLA-DR53, provenientes de diversas procedências, como France-Transplant (França), Pel Freez (EUA), Gene Trak (EUA) e Fred Hutchinson Cancer Research Center (EUA).

\subsection{Análise estatística}

As comparações das frequiências dos antígenos de histocompatibilidade entre os diversos grupos de indivíduos normais foram realizadas, utilizando-se o teste exato de Fisher, bicaudal $^{(10)}$, sendo considerada significante a possibilidade de ocorrência causal das diferenças observadas, quando não excedia $5 \%$, ou seja, $\mathrm{p}<0,05$.

\section{RESULTADOS}

\subsection{Antígenos HLA de classe I}

As freqüências dos antígenos HLA de classe I (HLA-A, -B, -C) nos indivíduos dos grupos G1 e G2, considerados como um todo, isto é, independentemente da etnia, são mostradas nas Tabelas I e II. As Tabelas III, IV e V mostram as freqüências dos antígenos HLA-A, -B e -C nos indivíduos pertencentes ao grupo G2, destarte considerados como caucasóides, mulatos ou negros.

Considerando apenas os grupos $\mathrm{G} 1$ e G2 como um todo, o antígeno HLA-A2 foi o mais freqüentemente encontrado, atingindo $46 \%$ em G1 e $50 \%$ em G2. A comparação da frequiência desse antígeno entre esses grupos não revelou diferença significante. As análises das frequiências dos outros antígenos HLA-A, observadas nos grupos G1 e G2 foram, a grosso modo, semelhantes, não sendo detectadas diferenças significantes na maioria das comparações realizadas. No entanto, as freqüências dos antígenos HLA-A23 e HLA-A26 estavam mais elevadas no grupo G1 em comparação ao G2.

As análises visuais das freqüências dos antígenos HLA-B entre os grupos G1 e G2 também mostram valores muito semelhantes, sendo que as comparações entre as freqüências desses antígenos não revelaram diferenças significantes. De modo análogo, as análises das frequiências dos antígenos HLA$\mathrm{C}$ entre os dois grupos não revelaram diferenças significantes, exceto em relação ao antígeno HLA$\mathrm{Cw} 2$, cuja freqüência estava, significantemente, mais elevada no grupo G1 ( $p<0,001)$. 
Tabela I - Freqüência (\%) dos antígenos HLA de classe I (HLA-A, -B, -Cw) e de classe II (HLA-DR) em indivíduos normais, do grupo G1. O sinal diacrítico (-), seguido da designação dos loci HLA, significa o número de "blanks", isto é, antígenos não definidos pelos anti-soros utilizados

\begin{tabular}{|c|c|c|}
\hline $\begin{array}{c}\text { HLA -A } \\
(n-100)\end{array}$ & $\begin{array}{l}\text { HLA -B } \\
(n-100)\end{array}$ & $\begin{array}{c}\text { HLA -Cw } \\
(\mathrm{n}-100)\end{array}$ \\
\hline$A 1-21$ & B7 -14 & Cw1 - 23 \\
\hline A2 -46 & B8 -21 & Cw2 - 23 \\
\hline A3 -12 & $\mathrm{~B} 13-10$ & Cw3 - 18 \\
\hline A11- 16 & B14 - 14 & Cw4 - 30 \\
\hline A23 - 18 & B15 - 02 & Cw5 - 12 \\
\hline A24-09 & B17 - 02 & Cw6 - 20 \\
\hline A25 - 13 & B18 - 20 & Cw7 - 27 \\
\hline A26 - 14 & B22 - 0 & Cw8 - 02 \\
\hline A28 - 10 & B27 - 02 & Cw- -45 \\
\hline A29 - 10 & B35 - 24 & \\
\hline A30 - 04 & B37 - 01 & \\
\hline A31 - 04 & $\mathrm{~B} 40-0$ & \\
\hline A32 - 05 & B50 - 0 & \\
\hline A33-02 & B38 - 1 & \\
\hline A34 - 0 & В39 - 0 & \\
\hline A35 - 0 & B44 - 16 & \\
\hline \multirow[t]{5}{*}{$A_{-}-16$} & B45 - 0 & \\
\hline & B49 - 0 & \\
\hline & B51 - 14 & \\
\hline & B52 - 01 & \\
\hline & B $-\quad-58$ & \\
\hline
\end{tabular}

\subsection{Antígenos HLA de classe II}

As freqüências dos antígenos HLA-DR nos grupos G1 e G3, considerados como um todo, são mostradas na Tabela VI. Ainda, naquela tabela, estão incluídas as frequiências desses antígenos no grupo G3, destarte subdivididos nas categorias caucasóides, mulatos e negros.

As freqüências dos antígenos HLA-DQ no grupo G3, considerado como um todo ou subdividido de acordo com o perfil étnico, são mostradas na Tabela VII.
Tabela II - Freqüência dos antígenos HLA de classe I (HLA-A, -B, -Cw) nos indivíduos-controle do grupo G2. As cifras entre parênteses indicam os números relativos (\%) de indivíduos tipificados para cada antígeno

\begin{tabular}{|c|c|c|c|c|c|}
\hline \multicolumn{2}{|r|}{$\begin{array}{l}\text { HLA-A } \\
(n-257)\end{array}$} & \multicolumn{2}{|r|}{$\begin{array}{l}\text { HLA-B } \\
(n-257)\end{array}$} & \multicolumn{2}{|r|}{$\begin{array}{l}\text { HLA-C } \\
(n-257)\end{array}$} \\
\hline $\mathrm{A} 1$ & - 50 (19) & B5 & - $12(5)$ & Cw1 & - $61(24)$ \\
\hline $\mathrm{A} 2$ & $-128(50)$ & B7 & $-40(16)$ & Cw2 & - $18(7)$ \\
\hline A3 & $-42(16)$ & B8 & $-29(11)$ & Cw3 & - $58(23)$ \\
\hline A9 & $-16(6)$ & B12 & $-\quad 3(1)$ & Cw4 & - $54(21)$ \\
\hline A10 & - $12(5)$ & B13 & - $14(5)$ & Cw5 & - $56(22)$ \\
\hline A11 & $-21(8)$ & B14 & $-21(8)$ & Cw6 & - $61(24)$ \\
\hline A19 & - $23(9)$ & B15 & $-14(5)$ & Cw7 & - 100 (39) \\
\hline A23 & - $17(7)$ & B16 & $-5(2)$ & Cw8 & - $21(8)$ \\
\hline A24 & $-45(18)$ & B17 & $-16(6)$ & & \\
\hline A25 & $-15(6)$ & B18 & $-29(11)$ & & \\
\hline A26 & $-3(1)$ & B21 & $-5(2)$ & & \\
\hline A28 & - $46(18)$ & B22 & $-\quad 1(0,4)$ & & \\
\hline A29 & $-17(7)$ & B27 & $-10(4)$ & & \\
\hline A30 & $-11(4)$ & B35 & - $49(19)$ & & \\
\hline A31 & $-3(1)$ & B37 & - $8(3)$ & & \\
\hline A32 & - $4(2)$ & B38 & $-11(4)$ & & \\
\hline A33 & $-6(2)$ & B39 & - $11(4)$ & & \\
\hline A34 & $-3(1)$ & B40 & - $8(3)$ & & \\
\hline A36 & $-5(2)$ & B41 & $-2(1)$ & & \\
\hline A68 & $-5(2)$ & B42 & $-\quad 1(0,4)$ & & \\
\hline A69 & $-3(1)$ & B44 & $-56(22)$ & & \\
\hline & & B45 & $3(1)$ & & \\
\hline & & B47 & - $2(1)$ & & \\
\hline & & B49 & $-16(6)$ & & \\
\hline & & B50 & $-2(1)$ & & \\
\hline & & B51 & - $49(19)$ & & \\
\hline & & B52 & $-\quad 4(2)$ & & \\
\hline & & B53 & $-5(2)$ & & \\
\hline & & B54 & $-\quad 1(0,4)$ & & \\
\hline & & B55 & $-\quad 1(0,4)$ & & \\
\hline & & B57 & $-13(5)$ & & \\
\hline & & B58 & $-6(2)$ & & \\
\hline & & $\mathrm{B} 60$ & $-16(6)$ & & \\
\hline & & $\mathrm{B} 62$ & $-12(5)$ & & \\
\hline & & B63 & $-\quad 1(0,4)$ & & \\
\hline
\end{tabular}




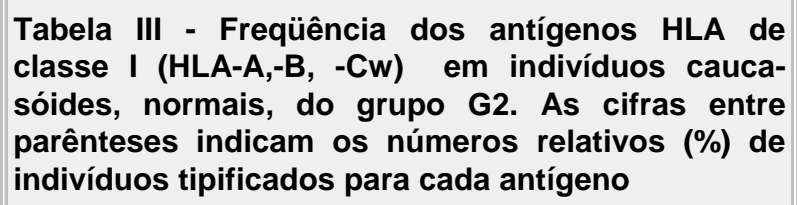
classe I (HLA-A,-B, -Cw) em indivíduos caucasóides, normais, do grupo G2. As cifras entre parênteses indicam os números relativos (\%) de indivíduos tipificados para cada antígeno

\begin{tabular}{|c|c|c|}
\hline $\begin{array}{l}\text { HLA -A } \\
(n-212)\end{array}$ & $\begin{array}{l}\text { HLA -B } \\
(n-212)\end{array}$ & $\begin{array}{l}\text { HLA -Cw } \\
(n-212)\end{array}$ \\
\hline 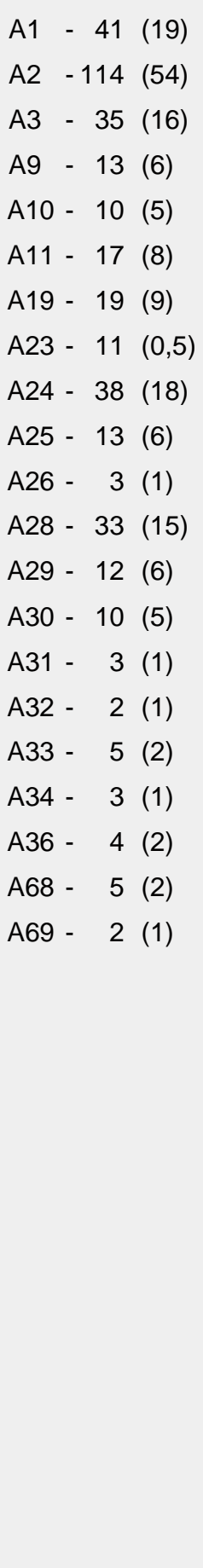 & $\begin{array}{lll}\text { B5 } & -11 & (5) \\
\text { B7 } & -34 & (16) \\
\text { B8 }-22 & (10) \\
\text { B12 - } & 3 & (1) \\
\text { B13 - } 11 & (5) \\
\text { B14 - } 18 & (8) \\
\text { B15 - } 11 & (5) \\
\text { B16 - } & 5 & (2) \\
\text { B17 - } 14 & (6) \\
\text { B18 - } 18 & (8) \\
\text { B21 - } & 4 & (2) \\
\text { B22 - } & 1 & (0,5) \\
\text { B27 - } & 7 & (3) \\
\text { B35 - } 36 & (17) \\
\text { B37 - } & 6 & (3) \\
\text { B38 - } & 9 & (4) \\
\text { B39 - } & 9 & (4) \\
\text { B40 - } & 6 & (3) \\
\text { B41 - } & 2 & (1) \\
\text { B42 - } & 1 & (0,5) \\
\text { B44 - } 48 & (23) \\
\text { B45 - } 2 & (1) \\
\text { B47 - } 2 & (1) \\
\text { B49 - } 14 & (6) \\
\text { B50 - } 2 & (1) \\
\text { B51 - } 41 & (9) \\
\text { B52 - } & 4 & (2) \\
\text { B53 - } & 4 & (2) \\
\text { B54 - } & 1 & (0,5) \\
\text { B55 - } & 1 & (0,5) \\
\text { B57 - } 12 & (6) \\
\text { B58 - } & 6 & (3) \\
\text { B60 - } 12 & (6) \\
\text { B62 - } 12 & (6) \\
\text { B63 - } & 1 & (0,5)\end{array}$ & $\begin{array}{l}\text { Cw1 - } 52(24) \\
\text { Cw2 - } 15(7) \\
\text { Cw3 - } 53(25) \\
\text { Cw4 - } 39(8) \\
\text { Cw5 - } 49(23) \\
\text { Cw6 - } 57(27) \\
\text { Cw7 - } 82(39) \\
\text { Cw8 - } 25(12)\end{array}$ \\
\hline
\end{tabular}

Tabela IV - Freqüência dos antígenos HLA de classe I (HLA-A,-B,-Cw) em indivíduos mulatos, do grupo G2. As cifras entre parênteses indicam os números relativos (\%) de indivíduos tipificados para cada antígeno

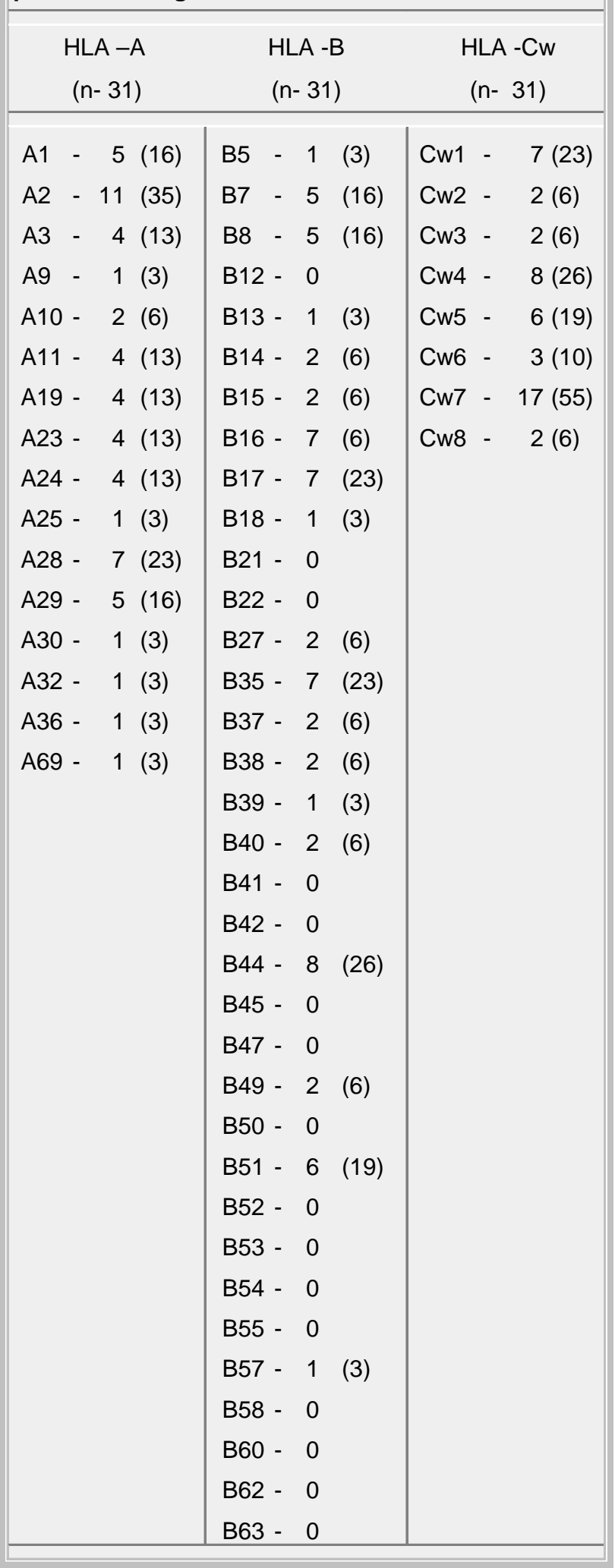




\begin{tabular}{|c|c|c|c|}
\hline \multicolumn{4}{|c|}{$\begin{array}{l}\text { Tabela V- Freqüência dos antígenos HLA de classe } \\
\text { I (HLA- A,-B,Cw) em indivíduos negros normais do } \\
\text { grupo G2. As cifras entre parêntesis indicam os } \\
\text { números relativos (\%) de indivíduos tipificados } \\
\text { para cada antígeno }\end{array}$} \\
\hline $\begin{array}{c}\text { HLA -A } \\
(n-12)\end{array}$ & & $\begin{array}{l}\text { HLA -B } \\
(n-12)\end{array}$ & $\begin{array}{c}\text { HLA -Cw } \\
(n-12)\end{array}$ \\
\hline $\begin{array}{lll}\text { A1 - } & 4 & (33) \\
\text { A2 - } & 2 & (16) \\
\text { A3 - } & 3 & (25) \\
\text { A9 - } & 2 & (16) \\
\text { A10 - } & 0 & (0) \\
\text { A11 - } & 0 & (0) \\
\text { A19 - } & 0 & (0) \\
\text { A23 - } & 1 & (8) \\
\text { A24 - } & 3 & (25) \\
\text { A28 - } & 5 & (42) \\
\text { A32 - } & 1 & (8) \\
\text { A33 - } & 1 & (8)\end{array}$ & $\begin{array}{l}\text { B5 } \\
\text { B7 } \\
\text { B8 } \\
\text { B12 } \\
\text { B13 } \\
\text { B14 } \\
\text { B15 } \\
\text { B16 } \\
\text { B17 } \\
\text { B18 } \\
\text { B21 } \\
\text { B22 } \\
\text { B27 } \\
\text { B35 } \\
\text { B37 } \\
\text { B38 } \\
\text { B39 } \\
\text { B40 } \\
\text { B41 } \\
\text { B42 } \\
\text { B44 } \\
\text { B45 } \\
\text { B47 } \\
\text { B49 } \\
\text { B50 } \\
\text { B51 } \\
\text { B52 } \\
\text { B53 } \\
\text { B54 } \\
\text { B55 } \\
\text { B57 } \\
\text { B58 } \\
\text { B60 } \\
\text { B62 } \\
\text { B63 }\end{array}$ & $\begin{array}{ll}- & 0 \\
- & 1(8) \\
- & 2(16) \\
- & 0 \\
- & 2(16) \\
- & 1(8) \\
- & 1(8) \\
- & 0 \\
- & 0 \\
- & 1(8) \\
- & 0 \\
- & 0 \\
- & 1(8) \\
- & 6(50) \\
- & 0 \\
- & 0 \\
- & 1(8) \\
- & 0 \\
- & 0 \\
- & 0 \\
- & 0 \\
- & 0 \\
- & 0 \\
- & 0 \\
- & 1(8) \\
- & 0 \\
- & 0 \\
- & 1 \\
- & 0 \\
- & 0 \\
- & 0 \\
- & 0 \\
- & 3(25) \\
- & 0 \\
- & 0\end{array}$ & $\begin{array}{ll}\text { Cw1 }-0 & \\
\text { Cw2 }-1 & (8) \\
\text { Cw3 }-2 & (16) \\
\text { Cw4 }-7 & (58) \\
\text { Cw5 - 1 } & (8) \\
\text { Cw6 - 1 } & (8) \\
\text { Cw7 }-1 & (8) \\
\text { Cw8 - 1 } & (8)\end{array}$ \\
\hline
\end{tabular}

\section{DISCUSSÃO}

A definição de indivíduo normal para estudos imunogenéticos tem sido associada à ausência de doença por ocasião da seleção para tais estudos. Contudo, esse tipo de abordagem pode sofrer várias críticas, como, por exemplo, os indivíduos saudáveis hoje podem apresentar algum tipo de doença amanhã. Nos inúmeros estudos já disponíveis na literatura universal, diversos grupos de indivíduos têm sido utilizados como controles para a avaliação do perfil imunogenético de uma determinada população. Os indivíduos saudáveis, pertencentes ao grupo de funcionários da instituição onde a pesquisa está sendo realizada, os doadores de sangue e os doadores de órgãos sólidos ou de células hematopoiéticas têm sido tradicionalmente considerados como representativos para os estudos das freqüências dos marcadores imunogenéticos. Particularmente, os estudos das freqüências dos antígenos de histocompatibilidade entre os doadores cadáveres de órgãos sólidos e os doadores vivos de células hematopoiéticas são bastante informativos, pois esses grupos, usualmente, são muito grandes e bastante representativos do perfil imunogenético da população em estudo $^{(11)}$. Assim, neste estudo, nos propusemos a avaliar o perfil imunogenético de três grupos de indivíduos considerados saudáveis, analisando as características de cada grupo individualmente, e, também, as comparações entre esses grupos.

Os estudos acerca das freqüências dos antígenos HLA na população brasileira são escassos ${ }^{(3 / 7)}$. O Brasil é um país de dimensões continentais, cuja população é caracterizada por uma grande miscigenação de indivíduous de diversos grupos raciais, predominando caucasóides, negros, índios e um vasto grupo de indivíduos oriundos das misturas entre esses grupos. Uma vez que a formação e distribuição desses indivíduos tenha características particulares, que possam variar de acordo com a região estudada, a tipificação dos marcadores de histocompatibilidade em cada região é de grande importância para os estudos de evolução, para uma seleção adequada de doadores em transplantes de órgãos sólidos ou células hematopoiéticas, e, ainda, para os estudos de associação com as doenças. Em estudo prévio, avaliando o polimorfismo dos antígenos HLA de classe I (HLA-A, -B e -C) em cinco populações brasileiras, provenientes das seguintes capitais: Porto Alegre, Curitiba, São Paulo, Rio de Janeiro e Goiânia, Trachtemberg et al., 1988, observaram mais similaridades, em termos de frequiências dessas especificidades, do que dissimilaridades ${ }^{(3)}$. Dois outros estudos, realizados na cidade de São Paulo, englobando um grande número de indivíduos normais, também mostram muitas semelhanças nas freqüências dos antígenos HLA de classe $\mathrm{I}^{(4,6)}$, sendo salientado que o perfil imunogenético dessas populações miscigenadas está mais próximo do pólo caucasóide do que do pólo negróide ${ }^{(4)}$. Recentemente, Moraes \& Moraes, 1996, estudaram um grande número de indivíduos normais, procedentes de diferentes regiões geográficas, dos estados de São Paulo, Rio de Janeiro, Paraná, Rio Grande do Sul, Pernambuco, Goiás, Minas Gerais e Ceará, tendo, também, verificado grande variabilidade alélica nesses indivíduos ${ }^{(5)}$. 
Tabela VI - Freqüência dos antígenos HLA-DR nos indivíduos do grupo G1 (n-100), nos indivíduos do grupo G3, considerados como um todo (n-191), ou separados como caucasóides (n-151), mulatos (n- 23) ou negros $(n-17)$. As cifras entre parênteses indicam os números relativos $(\%)$ de indivíduos tipificados para cada antígeno. $O$ sinal diacrítico (-), utilizado após o loci DR, indica o número de vezes em que a especificidade não foi definida pelo painel de anti-soros utilizado

\begin{tabular}{|c|c|c|c|c|c|c|c|c|c|}
\hline \multirow{2}{*}{\multicolumn{2}{|c|}{$\begin{array}{c}\mathrm{G} 1 \\
(\mathrm{n}-100)\end{array}$}} & \multicolumn{8}{|c|}{ G3 } \\
\hline & & \multicolumn{2}{|r|}{$(n-191)$} & \multicolumn{2}{|c|}{$(n-151)$} & \multicolumn{2}{|r|}{$(n-23)$} & \multicolumn{2}{|c|}{$(n-17)$} \\
\hline DR1 & -26 & DR1 & $-46(24)$ & DR1 & $-31(20)$ & DR1 & $-4(17)$ & DR1 & $-6(35)$ \\
\hline DR2 & -31 & DR2 & - $50(26)$ & DR2 & - 41 (27) & DR2 & - $5(22)$ & DR2 & $-\quad 4(23)$ \\
\hline DR3 & -28 & DR3 & $-46(24)$ & DR3 & - $39(25)$ & DR3 & $-5(22)$ & DR3 & $-3(17)$ \\
\hline DR4 & -23 & DR4 & - 70 (37) & DR4 & $-36(23)$ & DR4 & $-8(35)$ & DR4 & $-7(41)$ \\
\hline DR5 & -20 & DR5 & - $14 \quad(7)$ & DR5 & - $12(8)$ & DR5 & - 1 (4) & DR5 & $-1(5)$ \\
\hline DR6 & -06 & DR6 & - $14 \quad(7)$ & DR6 & - $12(8)$ & DR6 & - 1 (4) & DR6 & $-1(5)$ \\
\hline DR7 & -21 & DR7 & $-26(14)$ & DR7 & - 19 (12) & DR7 & $-5(22)$ & DR7 & $-1(5)$ \\
\hline DR8 & -02 & DR8 & - $3(2)$ & DR8 & - $3(2)$ & DR8 & $\begin{array}{lll}- & 0 & (0)\end{array}$ & DR8 & $-0(0)$ \\
\hline DR9 & -02 & DR9 & -10 & DR9 & - $23(15)$ & DR9 & $-3(13)$ & DR9 & $-0(0)$ \\
\hline DR10 & -0 & DR10 & - 1 (1) & DR10 & - $\quad 1(0,6)$ & DR10 & $\begin{array}{lll}- & 0 & (0)\end{array}$ & DR10 & - $0(0)$ \\
\hline \multirow[t]{6}{*}{$\mathrm{DR}_{-}$} & -41 & DR11 & $-43(23)$ & DR11 & $-24(15)$ & DR11 & $-4(17)$ & DR11 & $-3(17)$ \\
\hline & & DR12 & - 1 (1) & DR12 & $\begin{array}{lll}- & 0 & (0)\end{array}$ & DR12 & $\begin{array}{lll}- & 0 & (0)\end{array}$ & DR12 & - $0(0)$ \\
\hline & & DR13 & $\begin{array}{lll} & 17\end{array}$ & DR13 & - $\quad 3(2)$ & DR13 & $\begin{array}{lll}- & 0 & (0)\end{array}$ & DR13 & - $0(0)$ \\
\hline & & DR14 & - $11(6)$ & DR14 & - 7 (4) & DR14 & $-1(4)$ & DR14 & $-3(17)$ \\
\hline & & DR52 & $-143(75)$ & DR52 & $-103(67)$ & DR52 & $-16(70)$ & DR52 & $-13(76)$ \\
\hline & & DR53 & $-100(52)$ & DR53 & - $89(58)$ & DR53 & $-14(60)$ & DR53 & $-7(41)$ \\
\hline
\end{tabular}

\begin{tabular}{|c|c|c|c|c|}
\hline $\begin{array}{c}\text { HLA -DQ } \\
(\mathrm{n}-191)\end{array}$ & $\begin{array}{c}\text { HLA -DQ } \\
(n-151)\end{array}$ & $\begin{array}{c}\mathrm{HLA}-\mathrm{DQ} \\
(\mathrm{n}-23)\end{array}$ & $\begin{array}{r}\text { HLA -D } \\
(\mathrm{n}-17\end{array}$ & \\
\hline DQ1 - $125(65)$ & DQ1 - 103 (67) & DQ $1-17$ (73) & DQ1 - 9 & (52) \\
\hline DQ2 - $17 \quad(9)$ & DQ2 - $13 \quad(8)$ & DQ 2 - $2 \quad$ (9) & DQ2 - 2 & (11) \\
\hline DQ3 - 92 (48) & DQ3 - $81 \quad(53)$ & DQ $3-12$ (54) & DQ3 - 6 & (35) \\
\hline DQ4 - 1 & DQ4 - $0 \quad(0)$ & DQ 4 - $0 \quad(0)$ & DQ4 - 0 & (0) \\
\hline DQ7 - $55 \quad$ (29) & DQ7 - $40 \quad$ (26) & $\mathrm{DQ} 7-7$ & DQ7 - 6 & (35) \\
\hline
\end{tabular}

Com relação aos antígenos HLA de classe I, as maiores discrepâncias entre as freqüências dessas especificidades, nas diversas populações brasileiras, sem dúvida, se encontram nos antígenos HLA-A2. Nas populações de Porto Alegre, Curitiba, Rio de Janeiro e Goiânia, a frequiência desse antígeno atinge cerca de
$30 \%{ }^{(3)}$. Por outro lado, na população da cidade de São Paulo, os estudos mostram resultados bastante discrepantes, conforme o laboratório de histocompatibilidade. Os estudos de Gorodezki et al. ${ }^{(6)}$ relatam frequiências em torno de $25 \%$, ao passo que os de Rosales et al. ${ }^{(4)}$ relatam freqüências em torno de $50 \%$. Recente- 
mente, Moraes \& Mores, 1966, estudando um grupo de 1768 indivíduos mestiços, provenientes de diversas regiões do país (cidades de São Paulo, São José do Rio Preto, Bauru, Campinas, Rio de Janeiro, Porto Alegre, Maringá, Londrina, Recife, Brasília, Belo Horizonte, Fortaleza, Ribeirão Preto e Goiânia) relatam que as freqüências dos antígenos HLA-A2 atingem cerca de $50 \%{ }^{(5)}$. Em nossos estudos, avaliando a população brasileira da região nordeste do Estado de São Paulo, abrangendo predominantemente a população da cidade de Ribeirão Preto, as cifras para esses antígenos atingem também os patamares de $50 \%$, nos três grupos de indivíduos normais avaliados. Recentemente, os estudos moleculares de determinação do polimorfismo dos antígenos HLA-A2 apontam, pelo menos, 30 alelos, codificando esses antígenos ${ }^{(12)}$, mostrando a grande diversidade de alelos para essa especificidade. Assim, seria interessante realizar a avaliação do polimorfismo desse grupo de alelos, em nível genômico, nas diversas populações brasileiras, para uma posterior análise das discrepância observadas em estudos sorológicos.

Com respeito aos antígenos HLA de classe II, os estudos publicados também são escassos, o mais abrangente deles, o de Moraes \& Moraes $^{(5)}$, avalia as frequiências dos antígenos HLA-DR e HLA-DQ em uma população mestiça, abrangendo 186 indivíduos. As comparações das freqüências dos antígenos HLADR obtidas por Moraes \& Moraes ${ }^{(5)}$ com aquelas obtidas neste estudo são difíceis de serem realizadas, devido aos diferentes painéis de anti-soros utilizados e às mudanças das nomenclaturas dos alelos de classe
II. No intervalo de tempo (cerca de 15 anos) em que as tipificações das especificidades HLA de classe II foram realizadas neste estudo, muitas mudanças ocorreram nas definições desses antígenos pelo Comitê Internacional de Nomenclatura dos Antígenos HLA, em termos de antígenos amplos, como HLA-DR2, HLA-DR5, HLA-DR6 e HLA-DQ1, e antígenos privados, isto é, antígenos HLA-DR15 e DR16 ("splits" ou subtipos do antígeno amplo HLA-DR2), antígenos HLA-DR13 e DR14 (subtipos do HLA-DR6), antígenos HLA-DQ5 e DQ6 (subtipos do HLA-DQ1). Apesar disso, as comparações das frequiências das especificidades, que não foram subdivididas (HLA-DR1, HLA-DR4 e HLA-DR7), entre os grupos G1 e G3 deste estudo com aquelas relatadas por Moraes e Moraes (1996) mostram resultados semelhantes. Nas comparações das freqüências das especificidades HLA-DQ, conforme observadas no grupo G3 deste estudo, com aquelas relatadas por Moraes \& Moraes (1996), os mesmos comentários em relação aos antígenos HLADR podem ser feitos.

Considerando apenas os grupos avaliados neste estudo, verificamos que as frequiências dos antígenos HLA de classe I e II são, a grosso modo, semelhantes nos três grupos estudados, mostrando que os três grupos são representativos, em termos de perfil imunogenético, da população da região nordeste do Estado de São Paulo. Assim, as frequiências desses antígenos, avaliadas em quaisquer dos grupos aqui analisados, podem ser utilizadas em estudos populacionais, na seleção de doadores em transplantes e nos estudos de associação dos antígenos HLA com doenças.

DONADI EA; MAURÍCIO-DA-SILVA L; PAULA-SANTOS CM; SILVEIRA RD; DEGHAIDE NHS; FERRAZ AS \& VOLTARELLI JC. Frequency of histocompatibity antigens in the normal population of the northeast region of the state of São Paulo Brazil . Medicina, Ribeirão Preto, 33: 19-26, jan./march 2000.

ABSTRACT: Type of study: prevalence study.

Objectives: to evaluate the frequency of class I and class II histocompatibity antigens in the normal population of the Northeast region of the State of São Paulo, Brazil.

Methods: three groups of healthy individuals were studied: the staff personnel of the University Hospital and School of Medicine of Ribeirão Preto; local blood donors, and the cadaveric donors for solid organ transplantation. HLA antigens were typed by a complement-dependent microlymphocytotoxicity assay.

Results: HLA frequencies among individulas from the 3 groups sutudied here were similar.

Conclusions: HLA frequency observed in any of these populations may be used for donor selection purposes and for HLA and disease association studies.

UNITERMS: HLA Antigens. Serology. Histocompatibility Antigens Cass I. Histocompatibility Antigens Cass II. 


\section{REFERÊNCIAS BIBLIOGRAFICAS}

1 - BODMER W. HLA polymorphism: Origin and maintenance. In: TERASAKI PI \& GJERTSON DW, eds. HLA 1997, UCLA Tissue Typing Laboratory, Los Angeles, p. 1-6, 1997.

2 - BODMER J. HLA and anthropology. In: TERASAKI PI \& GJERTSON DW, eds. HLA 1997, UCLA Tissue Typing Laboratory, Los Angeles, p. 45- 59, 1997.

3 - TRACHTENBERG A; JOBIM LFJ; KRAEMER E; SALZANO FM; MORAES ME; MORAES JR; GERBASE-DE-LIMA M; ARCEGOMEZ B \& FERREIRA E. The HLA polymorphism in five Brazilian populations. Ann Hum Biol 15: 213-221, 1988.

4 - ROSALES T; GUILHERME L; CHIARELLA J; MARIN ML; ROSALES C; MELO CP; GOLDBERG AC \& KALIL J. Human leukocyte $A$ and $B$ antigen, gene and haplotype frequencies in the population of the city of São Paulo in Brazil. Braz $\mathbf{J}$ Med Biol Res 25: 39-47, 1992.

5 - MORAES JRF \& MORAES MEH. Distribuição dos alelos HLA na população barasileira. Hematol Hemoter 1: 18-23, 1996.

6 - GORODEZKY C; LOON J; MOLITERNO R; TORRES E; GERBADE-DE-LIMA M; PELEGRINO J; FERREIRA E; ALVAREZ I; CARRANZA JM; HERNANDEZ O; DE LA ROSA G; DEBAZ H; OLIVO A; MORENO M; LAYRISSE Z \& ELIAS M. HLA in some Latin American populations: Mexicans, Brazilian, Venezuelans, and Uruguayans. In: TSUJI K; AIZAWA M \& SASAZUKI T, EDS. HLA 1991. In: Proceedings XI International Histocompatibility Workshop and Conference. Oxford Science Publication, Oxford, p. 662- 665, 1992.
7 - IMANISHIT; AKAZAT; TOKUNAGAK \& GOJOBORIT. Allele and haplotype frequencies for HLA and complement loci in various ethnic groups. In: TSUJI K; AIZAWA M \& SASAZUKI T, eds. HLA 1991. Proceedings XI International Histocompatibility Workshop and Conference. Oxford Science Publication, Oxford, p. 1065 - 1220, 1992.

8 - MORAES ME; FERNANDEZ-VIÑA M; SALATIEL I; TSAI S; MORAES JR \& STATSNY P. HLA class II DNA typing in two Brazilian populations. Tissue Antigens 41: 238-242, 1993.

9 - TERASAKI PI \& MCCLELLAND JD. Microdrplet assay of human serum cytotoxins. Nature 204: 998-1000, 1964.

10 - SVEJGAARD A \& RUDER LP. HLA and disease association: Detecting the strongst association. Tissue Antigens 43: 1827,1994

11 - DYER P \& WARRENS A. Design and interpretation of studies of major histocompatibility complex in disease. In: LECHLER R, ed. HLA \& disease. Academic Press, London, p. 93-121, 1994.

12 - BODMER JG; MARSH SGE; ALBERT ED; BODMER WF; BONTROP RE; DUPONT B; ERLICH HA; HANSEN JA; MACH B; MAYR WR; PARHAM P; PETERSDORF EW; SASAZUKI T; SCHREUDER GMTH; STROMINGER JL; SVEJGAARD A \& TERASAKI PI. Nomenclature for factors of the HLA system, 1998. Tissue Antigens 53: 407-446, 1999.

Recebido para publicação em 14/01/2000

Aprovado para publicação em 03/03/2000 